\title{
Comparative Studies on the Visual Way of European Landscape and Chinese Landscape
}

\author{
Fang Li \\ School of Arts, Xi'an University, Xi'an 710065, China. \\ 88664410@qq.com
}

\begin{abstract}
Keywords: European landscape; Chinese landscape; Monet and Guo Xi; Visual way; comparative studies.

Abstract. This article has analyzed the value of the comparative studies on visual way of European landscape and Chinese landscape. It points out that the European landscape and Chinese landscape have their own views and the intrinsic value as to independent systems, and they have the status, role and contribution to the common culture in the world. Meanwhile, it expounds the phenomenon that European landscape and Chinese landscape learn from the other side to change in the collision and conversation.
\end{abstract}

\section{Introduction}

This article explores the spiritual origins and similarities and differences of European landscape and Chinese landscape, stressed on the point of 'visual way' combined with the examples of Monet and Guo Xi. By these to understand the context of their own development and evolving track much more thoroughly, and understand their intrinsic value properly. Apart from these, we can also get a good comprehension about the 'mutual understanding' and 'mutual complementary' between Chinese and Western artistic. So, it has a theoretical value and practical significance for the integration of the Chinese and Western in the new era.

\section{Visual Way}

\subsection{Interpretation of the Visual Way.}

In $\mathrm{Ci} \mathrm{Hai}$, the meaning of vision is 'vision is the sense to feel and distinguish the brightness, color and other characteristics of light'. In painting, the meaning of visual way is that the artists have understood the observation purposes, tasks and objects clearly, and then to 'see what they had seen' spontaneously according to their aesthetic tastes and the requirements of their artistic expression. Through this aesthetic observation, artists can get a sharp and clear perceptual impression about the visual objects.

\subsection{Visual Way in the European Landscape.}

Influenced by the thinking of 'Subject from Object Points', European landscape pay much attention to the human's management and transformation on the nature. During the process of painting, the artists treat the objects to draw in an objective and analytical attitude, study the external objective form of the objects and then pay more attention to imitate the body and color of them. Artists in the era of European Renaissance, they inherited the ancient Greek traditional theory directly, and they also largely affected by the humanistic ideas and natural sciences in their age, so they have researched on the perspective science, human anatomy, color science and the light shading method. This imitating the natural objects had extended to the era of Impressionist Painting Period.

\subsection{Visual Way in the Chinese Landscape.}

From the developing track of Chinese Landscape from its beginning in Jin and Song Dynasty to it became mature in the period of Five Dynasties and the Northern Song Dynasty, we can see the historical evolution of the Chinese Landscape's visual way. In Chinese Landscape, the "vision" is not the same as "look", it not only includes all of the "view", it even includes the "synaesthesia" and "thinking" that have something to do with the "vision" without any look. What it stressed on is not 
watch on the objects, is to get the true feeling of their heart, they stressed on the spiritual space, the mental observation and the psychological look.

\section{Method of Static Depth and Dynamic Moving Watching}

\subsection{Method of Static Depth.}

During the evolution process of European Landscape, no matter how many genres it had experienced, it always treats the true "form" and "color" of visual as its center, and this center had never changed. Although the Impressionist painter had led revolution to the language form of the oil painting, they also carried out this around the center of "visual reality". Therefore, the visual way of European Landscape is "visual image". European Landscape painters usually stand in a fixed time period, and a fixed position to appreciate the nature from a fixed point of view, this is a visual way of fixed point, fixed time and fixed phenomenon. This visual way usually takes the focus perspective and air perspective as its theoretical support. As far as the way of visual expression as concerned, it pursuit of the objective of the natural form, and the dynamic changes of image are solidified to a "flash", this makes European Landscape become an instant freeze-frame of real space, showing a real "method of space static depth" biased vision (Fig. 1).
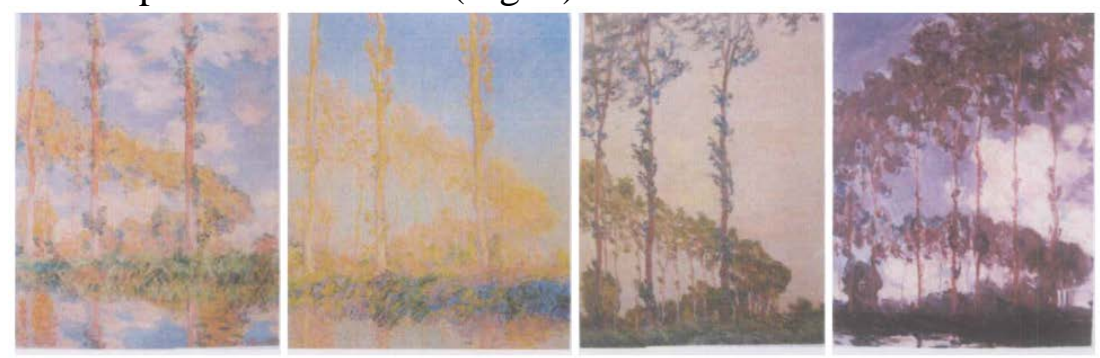

Fig.1 Monet · Les Peupliers

Monet is the representative of impressionist, and he is also an extreme impressionist. All of his life, he pursuit of the central thinking of the Impressionism, and try to capture the fleeting moment, reproduce his personal visual impression of the "moment", and depicting the moment of time changes. In one of the paintings Les Peupliers, the effect of light only lasted seven minutes, when the sunshine left a leaf, he took out another piece of canvas to paint. Due to the same landscape, he painted is not only a picture, but a group of pictures, so he is able to transfer the effect of one moment to another moment. His other series like Haystack and Rouen Cathedral, they can clearly reflect the changing of the seasons and the nature of a specific time in a day. As Fu Baoshi had said before, "Western painting is realistic, and describe the features of objects accurately based on the benchmarks of time and light”.

\subsection{Method of Dynamic Moving Watching.}

The most fundamental, significant visual way of Chinese Landscape, and throughout it all the way, we call it "the method of distant", and "the method of distant" is the soul of Chinese Landscape's visual way. The 'distant' in Chinese Landscape's "method of distant" is a kind of "point of view", it has many foothold of body, like: foot of the mountain, front of the mountain and close to the mountain; it also has the object to watch, such as the top of the mountain, the back of the mountain and the mountain far away (Fig. 2).

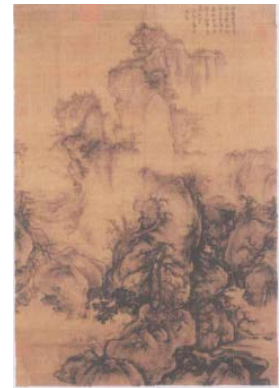

Fig.2 Guo Xi · ZaoChun Tu 


\section{Unify the Subject and Object, Integrating the Feeling and Scene}

\subsection{Unify the Subject and Object.}

Both Chinese Landscape and European Landscape reflect subjective feelings in varying degrees and express the spirit of the subject. Meanwhile, all of them has not avoid reproducing the shape of the objective image. Through this, they can get the dual harmony both between their mental world and their painting, and between their spirit and their painting. However, Chinese Landscape painters and the nature is a whole body, they are not only the observer of the nature, but also the participator. So, the "visual way" of both Chinese Landscape and European Landscape are all showing in the unification of subject and object. This we can find in Monet's Water Lilies and Guo Xi's ShuangSongGe Tu.

Feature of the "Objective"

Western culture takes the "subject-object separation" as the fundamental mode, "subject-object separation" is conscious of cosmic, this makes the western culture tends to put their thinking on the object world raising activities. In the aspect of artistic and aesthetic culture, we must pay much attention to imitate the nature, and they think the essence of art is imitation of nature. This acquirement of reality in the vision from the imitation of nature has been extended to the period of Impressionist painting.

Chinese Landscape painters also focus on the objective physical nature, feeling of images, observe and depict. In "Lin Quan Gao Zhi Ji”, Guo Xi had said that "Seasons are all different from each other, spring's harmony, summer's lush, autumn's sparse and thin and winter's dark”. He also pointed out that, no matter the water or the sky, their color is different all the year round. So, like the European Landscape, Chinese Landscape also has a perception and comprehension to the objective nature, but they use different way to express the color.

Feature of the "Subjective"

Arnheim had ever said, "visual image is never the mechanical reproduction of emotional material, but a creative grasp of the reality, the image it had grasped is rich in imagination, creativity, sensitivity of the image of the beauty”. During the progress of observing and painting in both Chinese and European Landscape, all of them want to express their spiritual life through their works. Chinese Landscape explain the nature through the way of "impressionistic", this has reflected the ideal and force of the personality, and it also has reflected the spiritual life of the painters. Although the European Landscape also focus on the "impressionistic", but it is not just imitation, they often reinterpret the nature during the progress of painting, and reveal a light of rationality, and by this, it also reflects the spiritual life of the painters. So, this is the subjective features of Chinese and European Landscape (Fig. 3 and Fig. 4).

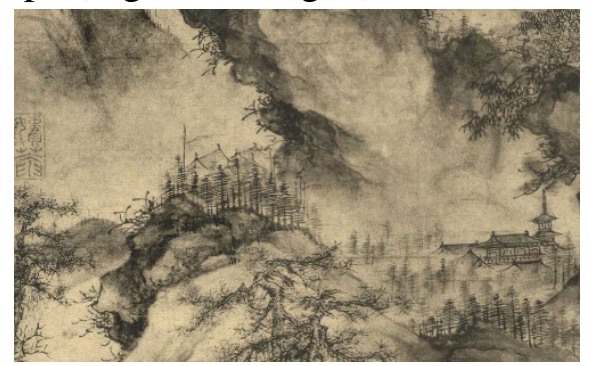

Fig.3 Monet · Water Lilies

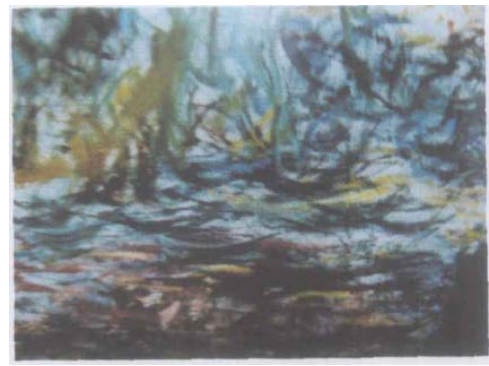

Fig.4 Guo Xi · ShuangSongGe Tu

\subsection{Integrating the Feeling and Scene.}

"Feeling" is the artist's subjective emotion, "scene" is a natural target object. The integrating of the feeling and scene is the aesthetic pursuit for both Chinese and European Landscape painters. In order to reveal the emotion in the natural scenery, we will change the target object as painters' emotion's change, and the visual way will also be changed. And the painters' emotion has been emerged into the nature scenery, and in the scenery, it is occupied by the emotion, so they will not just the ordinary scenery, they are a clever fusion of the emotion and the scene. This integrating of feeling and emotion in Chinese Landscape and European Landscape is revealed in the deeper structure 
through the external manifestations. This consists of the visual way in Monet and Guo Xi's landscape painting.

\section{Conclusion}

The $21^{\text {st }}$ Century has become a "globalization" era of comprehensive exchange, during the collision of the exchange of Chinese and Western artist, they stimulating each other, learning from each other, infiltrating into each other; nowadays is also abundant in the materials, the exchange of information can be carried out in a moment, and the life also become changing and fast, this is a new era different from all of the era before. The visual culture is extremely developed, all of the living environment and the way of life have changed obviously.

Under this circumstance, this article study the Chinese Landscape and European Landscape based on Monet and Guo Xi, and do our best to find out the similarity, difference and influence on each other. In order to better understand the development and evolution track of the Chinese and European Landscape painting. And understand the intrinsic value of them in an appropriate way, apart from this, we can also have a clear understanding on the "mutual understanding" and "complementary" between the Chinese Landscape and European Landscape.

\section{References}

[1] MADIHAFARAGMILLER, KEITHMILLER, JB.KIRKPATRICK. Determining the Accuracy of Historical Landscape Paintings [J]. Geographical Research, 2012, 511.

[2] Wei KONG. Imagery Scenery Innovative Expression of Washing Landscape Painting Photography [J]. Studiesin Sociology of Science, 2012, 33.

[3] Rahul Devrani, Vimal Singh. Determining the geomorphic changes in Srinagar (Garhwal) valley, NW Himalaya in last two centuries using landscape painting [J]. Zeitschriftfür Geomorphologie, NF, 2014, 582.

[4] Fred Myers. Emplacement and Displacement: Perceiving the Landscape Through Aboriginal Australian Acrylic Painting [J]. Ethnos, 2013, 784. 